\title{
OPRM1 and COMT polymorphisms: implications on postoperative acute, chronic and experimental pain after cardiac surgery
}

\author{
Maja Matic*,‡,1,2 (D), Sjoerd de Hoogd ${ }^{\ddagger 3} 3$, Saskia N de Wildt ${ }^{2,4}$, Dick Tibboel ${ }^{2}$, Catherijne AJ \\ Knibbe $^{3}$ \& Ron HN van Schaik ${ }^{1}$ \\ ${ }^{1}$ Department of Clinical Chemistry, Erasmus MC - University Medical Center Rotterdam, Wytemaweg 80, 3015 CN Rotterdam, \\ The Netherlands \\ 2Intensive Care \& Department of Paediatric Surgery, Erasmus MC - University Medical Center Rotterdam, Sophia Children's \\ Hospital, Wytemaweg 80, 3015 CN Rotterdam, The Netherlands \\ ${ }^{3}$ Department of Clinical Pharmacy, St Antonius Hospital, Koekoeklaan 1, 3435 CM Nieuwegein, The Netherlands \\ ${ }^{4}$ Department of Pharmacology \& Toxicology, Radboud University, Nijmegen, The Netherlands \\ *Author for correspondence: Tel.: +31 010703 8775; m.matic@erasmusmc.nl \\ $¥$ Authors contributed equally
}

\begin{abstract}
Aim: Investigate the potential role of OPRM1 (mu-opioid receptor) and COMT (catechol-Omethyltransferase enzyme) polymorphisms in postoperative acute, chronic and experimental thermal pain. Methods: A secondary analysis of 125 adult cardiac surgery patients that were randomized between fentanyl and remifentanil during surgery and genotyped. Results: Patients in the fentanyl group with the COMT high-pain sensitivity haplotype required less postoperative morphine compared with the averagepain sensitivity haplotype $(19.4[16.5 ; 23.0]$ vs $34.6[26.2 ; 41.4] ; p=0.00768)$, but not to the low-pain sensitivity group (30.1 $[19.1 ; 37.7] ; p=0.13)$. No association was found between COMT haplotype and other pain outcomes or OPRM1 polymorphisms and the different pain modalities. Conclusion: COMT haplotype appears to explain part of the variability in acute postoperative pain in adult cardiac surgery patients.
\end{abstract}

First draft submitted: 1 October 2019; Accepted for publication: 6 December 2019; Published online: 22 January 2020

Keywords: adverse drug reactions $\bullet$ drug transporter genes $\bullet$ pain

Adequate pharmacological management of pain is hampered by large variability between individuals in pain sensitivity and in analgesic response. Factors contributing to the extensive variability observed in pain and analgesia are considered multifactorial, including among other sex [1,2], age [3], race [4,5], co-medication (chronic opioid history/opioid addiction), co-morbidities [6,7], psychological elements such as anxiety [8] and genetic predisposition [9]. The need for a personalized approach, by means of genetics, environmental, psychological and injury-specific factors, has been acknowledged previously [10]. Moreover, chronic postsurgical pain also necessitates the need for an individualized pain management approach. By identifying patients at risk for this chronic postoperative pain state prior to surgery, healthcare providers could prevent its occurrence by adaptions in the pre-operative and postoperative treatment.

The genetic contribution in pain and pain treatment has been extensively studied the last two decades in the adult population by use of knock-down animal studies, twin studies, candidate gene approaches and genome-wide analyses [11,12]. The most widely studied and confirmed variant is the mu-opioid receptor (OPRM1) polymorphism $118 \mathrm{~A}>\mathrm{G}$, which has been associated with higher postsurgical opioid requirement in a recently performed metaanalysis [13]. The increased opioid requirement in $118 \mathrm{G}$ allele carriers was later confirmed in 1500 additional patients [14]. The 118G variant allele also relates with lower frequency of nausea and vomiting [14]. Both findings on opioid demand and adverse events are indicative for lower potency of exogenous opioids in carriers of the 118G allele.

Another important gene, repeatedly associated in studies with pain and pain treatment, is COMT, encoding the catechol-O-methyltransferase. The COMT rs4680 variant has been related with several phenotypes of pain [15] and opioid requirements [16-19]. Three different pain sensitivity haplotypes composed from this SNP and three other 
variants (rs4818, rs4633 and rs6269), being low-pain sensitivity (LPS), average-pain sensitivity (APS) and high-pain sensitivity (HPS) have been identified [20]. The pain sensitivity haplotypes have been attributed to the differences in COMT activity, with the LPS haplotype having 4.8-times higher activity compared with the APS and 11.4 to HPS haplotype [20]. COMT is responsible for the breakdown of catecholamines such as (nor)epinephrine and dopamine. Decreased COMT activity in rats has been associated with increased pain sensitivity via amplified firing at the $\beta_{2}$ and $\beta_{3}$-adrenergic receptors [21]. Altered dopamine levels in rats have been related with the expression of the endogenous opioid agonist, enkephalin [22]. Confirming this hypothesis, the COMT variant Val158Met (rs4680) in healthy adults, leading to a fourfold decrease in activity [23], reduced mu-opioid receptor density [24].

Up to date these genes have not been addressed across three different pain etiologies (acute and chronic postoperative pain and experimental pain) within the same individuals. The main aim of this genetic study was to assess if the highly investigated genetic variants in OPRM1 and COMT are related with acute postoperative pain reflected by postoperative opioid consumption and could predict the development of chronic postsurgical pain in cohort of patients undergoing cardiac surgery [25-27]. Additionally, the relation between these genetic variants and preoperative and postoperative thermal pain sensitivity has been assessed.

\section{Methods}

This is a candidate gene association study performed as a secondary analysis of a randomized clinical trial evaluating the effect of remifentanil versus fentanyl during cardiac surgery on the incidence of acute and chronic thoracic pain in the St Antonius Hospital in Nieuwegein, The Netherlands. The study received approval from the Regional Medical Ethical Review Board (Verenigde Commissies Mensgebonden Onderzoek R13.013) and was registered on the Clinical Trials register (ClinicalTrials.gov number NCT02031016). The study protocol has been published previously [26. Participants who signed written informed consent and from which a blood sample for DNA analysis was available were included in the current study. Since this is a secondary study, details about the primary clinical outcome and experimental pain thresholds can be found elsewhere $[25,27]$.

\section{Participants}

Inclusion criteria for the original study were age between 18 and 85 years, weight between 45 and $140 \mathrm{~kg}$ and planned cardiac surgery via sternotomy (i.e., elective coronary artery bypass grafting [CABG] and/or valve replacement). Exclusion criteria were pregnancy/breastfeeding, language barrier, history of drug abuse, chronic pain conditions (e.g., peripheral neuropathy, fibromyalgia), remifentanil/fentanyl/morphine/paracetamol allergy, BMI over $35 \mathrm{~kg} / \mathrm{m}^{2}$ and prior cardiac surgery.

\section{Study protocol}

In the original study, patients were randomized intraoperatively to either receive remifentanil continuous infusion (start $0.15 \mathrm{mcg} / \mathrm{kg}$ ideal bodyweight $/ \mathrm{min}$; adjusted when necessary) or extra fentanyl bolus (200-500 mcg), both on top of standard care with fentanyl bolus $(200-500 \mathrm{mcg}$ ) on predetermined times (prior, during sternotomy, during aorta cannulation and during opening pericardium). The attending anesthesiologist determined, based on patient's clinical monitoring (e.g., hemodynamics and sweating) and characteristics such as bodyweight or ejection fraction), the exact dose of fentanyl and whether additional fentanyl was required. Anesthesia induction was standardized in all patients with intravenous midazolam $(2.5-5.0 \mathrm{mg})$, followed by propofol bolus $(1-2 \mathrm{mg} / \mathrm{kg})$, pancuronium $(0.05-2 \mathrm{mg} / \mathrm{kg}$ ) and fentanyl (on time points as previously mentioned). 30 minutes before end of surgery all patients received 5-10 mg morphine intravenously.

Postoperative pain management after transfer to the ICU and the postanesthesia care unit included continuous morphine infusion (starting $2 \mathrm{mg} / \mathrm{h}$ ) and paracetamol 4-times daily (oral/intravenous). Adaption of the morphine infusion and/or additional morphine bolus doses was standardized and based on the numerical rating scale (NRS). The NRS was assessed three-times daily by the nurse or was selfreported if possible, based on a previously reported pain titration protocol [28]. An NRS > 4 was indicative for insufficient pain control. In the case the patient was not or insufficiently awake the nurse judged pain with NRS. From 24-h postoperatively onward, patients experiencing insufficient pain control despite dose escalation or side effects, continued receiving morphine boluses or were switched, at discretion of the attending physician, to oral oxycodone or tramadol. The development of chronic thoracic pain was identified with a questionnaire partly based on the validated Brief Pain Inventory [29], which was sent by an e-mail or post mail at 3, 6 and 12 months after surgery by the same researcher. Chronic thoracic pain was defined as sternal and/or thoracic pain (NRS $>0$ ) which the patient identified as related to surgery. 


\section{Quantitative sensory testing}

Cold and heat detection and pain thresholds were determined in the cardiac patients one day before, 3 days after and 12 months after surgery with the Method of Limits (MLI) by use of the Thermal Sensory Analyzer II 2001 (Medoc Advanced Medical Systems, Israel). The thermode $\left(30 \times 30 \mathrm{~mm}^{2}\right)$ was attached to the nondominant hand. Patients responded on the thermal stimuli by clicking a computer mouse with the dominant hand, when the detection or pain threshold was reached. Before formal determination of the thresholds started, a minimum of two training sessions with a test-retest difference below $20 \%$ was required. The thresholds were constructed by taking the average of four formal thresholds. Since the MLI method is depending on the reaction time of the individual the analysis with the thermal thresholds was corrected for median reaction time. Reaction time was determined with the open-source software (http://delphiforfun.org/Programs/Reaction_times.htm) by clicking the computer mouse in reaction to the appearance of a blue ball on a white screen. This response was rehearsed three-times and followed by five formal measurements, of which the mean reaction time was calculated per individual. All quantitative sensory testing tests in this study were performed by the same researcher $(\mathrm{SdH})$.

\section{Outcomes}

The outcomes studied in this candidate gene study were cumulative postoperative morphine requirements during the first $24 \mathrm{~h}(\mathrm{mg} / 24 \mathrm{~h})$ and $48 \mathrm{~h}(\mathrm{mg} / 48 \mathrm{~h})$; the development of chronic thoracic pain at 3, 6 and 12 months after surgery (yes/no); and thermal pain thresholds before surgery, 3 days and 12 months after surgery. Opioid requirements during first $48 \mathrm{~h}$ were calculated as morphine equivalents [30,31]. All outcomes were assessed for a relation with the genetic variant OPRM1 rs1799971, COMT rs4680, rs4818, rs4633, the COMT haplotype and the combined OPRM1 rs1799971/COMT rs4680 effect.

\section{Genotyping}

The genetic analysis was performed at the department of Clinical Chemistry at the Erasmus University Medical Centre in Rotterdam (The Netherlands). DNA was extracted from $1 \mathrm{ml}$ peripheral blood. DNA was isolated on the MagNA Pure LC 2.0 instrument (Roche ${ }^{\circledR}$, Almere, The Netherlands) with the 'DNA Isolation Kit Large volume' (Roche ${ }^{\circledR}$, Almere, The Netherlands). The 7500 Fast Real-Time PCR System (software version 3.0.0; Applied Biosystems, Bleiswijk, The Netherlands) was used for determination of the OPRM1 118A $>\mathrm{G}$ (rs1799971), COMT 472G >A (rs4680, rs4818 and rs4633) genetic variants with ready-made TaqMan ${ }^{\circledR}$ SNP Genotyping Assays. All single nucleotide polymorphisms were checked for agreement with minor allele frequency reported in literature and violation of Hardy-Weinberg equilibrium ( $\mathrm{p}$-value $>0.013$ ). $\mathrm{R}$ (version 3.1.1) haplo.stats package was used to estimate the COMT haplotype (posterior probability limit $>90 \%$ ). LPS group was encoded by the GGC (rs4680, rs4818, rs4633 resp.) haplotype, APS by ACT and HPS by GCC [32]. Participants with LPS/LPS and LPS/APS alleles were combined in the 'LPS' group, APS/APS and LPS/HPS in the 'APS' group and HPS/HPS and APS/HPS alleles in the 'HPS' group. Additionally, as previous findings indicate the combined effect of OPRM1 rs1799971 and COMT rs4680, we assessed this combined genotype [33,34], with group 1 represented by OPRM1 118AA genotype with COMT 472A allele carriage and group 2 by OPRM1 $118 \mathrm{G}$ allele carriage with/or COMT 472GG genotype.

\section{Statistical analysis}

IBM SPSS Statistics 21.0 was used for the statistical analysis. As previously reported [25], subjects that received continuous remifentanil infusion during surgery were predisposed to higher morphine consumption (median: $34.3 \mathrm{mg} / 24 \mathrm{~h}$ [interquartile range (IQR): $25.3 ; 48.2$ ]) postoperatively compared with the fentanyl randomization group $(30.2[19.2 ; 38.1])$. Due to this difference the cohort was stratified according to randomization group in the analysis between genetics and opioid consumption. After stratification the analysis with OPRM1 rs1799971 and combined OPRM1 rs1799971/COMT rs4680 genotype in relation to postoperative morphine requirement was performed with an Student's t-test. The analysis between COMT haplotype, COMT rs4680, rs4818 and rs4633 SNP with postoperative morphine requirement was calculated with the analysis of variance (ANOVA) test. A binominal logistic regression analysis was used in order to assess if the genetic variants, by adjusting for randomization arm (correction only made in analysis at 3 months) and age, could predict the likelihood of patients developing chronic thoracic pain at 3,6 and 12 months after surgery. The association with the MLI thermal thresholds has been corrected for the composite variable age and reaction time (FAC1_1) in a multiple linear 


\begin{tabular}{|c|c|c|c|}
\hline Demographic or clinical characteristics & Fentanyl $(n=63)$ & $\begin{array}{l}\text { Remifentanil } \\
(n=63)\end{array}$ & $\mathrm{p}$-value \\
\hline Gender (male/female) & $57 / 6$ & $58 / 5$ & 0.75 \\
\hline Age (years) & $66.1(7.6)$ & $62.1(9.0)$ & 0.007 \\
\hline BMI $\left(\mathrm{kg} / \mathrm{m}^{2}\right)$ & $28.0(3.1)$ & $27.5(3.6)$ & 0.47 \\
\hline $\begin{array}{l}\text { Ethnicity: } \\
\text { - Caucasian } \\
\text { - Asian }\end{array}$ & $\begin{array}{l}62 \\
1\end{array}$ & $\begin{array}{l}61 \\
2\end{array}$ & 0.99 \\
\hline Diabetes (yes/no) & $14 / 49$ & $10 / 53$ & 0.36 \\
\hline COPD (yes/no) & $4 / 59$ & $4 / 59$ & 0.99 \\
\hline Depression (yes/no) & $1 / 62$ & $4 / 59$ & 0.37 \\
\hline $\begin{array}{l}\text { Type of surgery: } \\
\text { - CABG } \\
\text { - Valve replacement } \\
\text { - Both }\end{array}$ & $\begin{array}{l}51 \\
9 \\
3\end{array}$ & $\begin{array}{l}49 \\
7 \\
7\end{array}$ & 0.40 \\
\hline Length hospital admission (days) & $5.0[3.0-7.0]$ & $5.0[3.0-7.0]$ & 0.67 \\
\hline Length ICU/PACU admission (hours) & $19.5[16.7-22.4]$ & $19.6[16.2-21.4]$ & 0.77 \\
\hline NRS (before surgery) & $0[0-0]$ & $0[0-0]$ & 0.77 \\
\hline Duration surgery (min) & $187(46.7)$ & $198(70.8)$ & 0.32 \\
\hline Length of anesthesia (min) & $219(49.0)$ & $233(72.1)$ & 0.18 \\
\hline Time to extubation ( $\mathrm{min}$ ) & $548[479-724]$ & $532[465-605]$ & 0.31 \\
\hline Intra-operative fentanyl (mg) & $2350[1750-3000]$ & $1750[1500-2500]$ & 0.001 \\
\hline Intraoperative remifentanil (mcg) & NA & $2165(696)$ & $<0.001$ \\
\hline Post-OK morphine consumption (mg/24 h) & $30.2[19.2-38.1]$ & $34.3[25.3-48.2]$ & 0.028 \\
\hline Post-OK opioid consumption (mg/48 h) & $39.0[26.2-51.4]$ & $46.8[33.8-59.2]$ & 0.047 \\
\hline Chronic thoracic pain 3 months after surgery (yes/no) & $21 / 42$ & $32 / 31$ & 0.047 \\
\hline Chronic thoracic pain 6 months after surgery (yes/no) & $20 / 42$ & $17 / 45$ & 0.56 \\
\hline Chronic thoracic pain 12 months after surgery (yes/no) & $12 / 49$ & $11 / 50$ & 0.82 \\
\hline
\end{tabular}

regression. The corrected mean and standard deviations of the thermal thresholds are displayed per genotype group, which have been retrieved with two-way analysis of covariance (ANCOVA).

Per outcome (e.g., thermal pain, morphine requirement, chronic pain) six analysis (OPRM1 rs1799971, COMT rs4680, rs4818, rs4633, COMT haplotype and combined OPRM1 rs1799971/COMT rs4680 genotype) were performed. Therefore, a two-sided p-value of $0.05 / 6=0.0083$ (Bonferroni correction) was considered statistically significant.

For the original study, a sample size calculation was performed on the primary end point chronic thoracic pain, and was based on the findings of a previous study [35]. This resulted in a total number of 117 patients, with a power of 0.80 and a two-sided significance level of 0.05 . Taking into account a mortality rate of $8 \% 1$-year after surgery [35], the total number of patients is 126 , which results in 63 subjects per arm.

\section{Results}

The original randomized controlled trial included 126 subjects (63 remifentanil/63 fentanyl) undergoing cardiac surgery at the St Antonius Hospital Nieuwegein. The cohort existed of mainly male individuals (91\%) and was almost completely of Caucasian origin $(98 \%)$, the remaining $2 \%(\mathrm{n}=3)$ had Asian descent. The most frequent cardiac procedure was CABG (79\%), followed by valve replacement (13\%) or patients having both procedures (8\%). In almost all CABG patients (98\%), the internal mammary artery (left, right or both) was used for coronary bypass. Totally, $56 \%$ of the patients was overweight (BMI: $25-29.9$ ), $24 \%$ moderately obese (BMI: 30-35) and 20\% had a normal BMI (18.5-24.9). For an overview of all demographic and clinical data according to randomization group see Table 1. One individual was excluded from further analysis due to a missing blood sample for DNA analysis. The selected genetic variants were in line with the frequencies reported in literature and did not deviate from the Hardy-Weinberg equilibrium (Table 2). 


\begin{tabular}{|c|c|c|c|c|c|}
\hline Genotype & Fentanyl & Remifentanil & MAF observed (\%) & MAF literature $^{\dagger}(\%)$ & HW equilibrium $p$-value \\
\hline OPRM1 (rs1799971) & & & 14 & 15 & 0.19 \\
\hline $118 \mathrm{AA}$ & 46 & 49 & & & \\
\hline $118 \mathrm{GG}$ & 1 & 3 & & & \\
\hline СОМТ (rs4680) & & & 51 & 48 & 0.99 \\
\hline $472 \mathrm{GG}$ & 17 & 13 & & & \\
\hline СОMT (rs4818) & & & 38 & 42 & 0.68 \\
\hline $408 \mathrm{CC}$ & 25 & 24 & & & \\
\hline $408 \mathrm{CG}$ & 24 & 33 & & & \\
\hline 408GG & 14 & 5 & & & \\
\hline СОMT (rs4633) & & & 51 & 48 & 0.99 \\
\hline $186 C C$ & 17 & 13 & & & \\
\hline $186 C T$ & 30 & 33 & & & \\
\hline
\end{tabular}

$\dagger$ NCBI SNP database (European population).

$\ddagger$ HW equilibrium for the total cohort $(n=125)$.

HW: Hardy-Weinberg; MAF: Minor allele frequency.

\section{Genetics versus acute postoperative pain}

After stratification according to the intraoperative randomization group, the COMT haplotype was related with postoperative morphine consumption $(\mathrm{mg} / 24 \mathrm{~h})$ within the intraoperative fentanyl group $(\mathrm{p}=0.009)$ but this was not the case for remifentanil $(\mathrm{p}=0.29)$. Post-hoc test (Bonferroni) correction confirmed that only the HPS haplotype group required significantly less morphine compared with the APS haplotype group (median $=19.4 \mathrm{mg} / 24 \mathrm{~h}[16.5-$ $23.0]$ vs $34.6 \mathrm{mg} / 24 \mathrm{~h}[\mathrm{IQR}=26.2-41.1] ; \mathrm{p}=0.007)$. No significant difference could be observed between the LPS haplotype $(30.1 \mathrm{mg} / 24 \mathrm{~h}[19.1-37.7])$ with APS ( $\mathrm{p}=0.13)$ or HPS ( $\mathrm{p}=0.15)$ haplotype groups. Total postoperative opioid consumption within $48 \mathrm{~h}$ postoperatively was also decreased in the COMT HPS haplotype $(\mathrm{p}=0.025)$, but no longer significant after Bonferroni correction. No associations were found between postoperative opioid consumption with OPRM1 rs1799971, the individual COMT SNPs (rs4680, rs4818 and rs4633) composing the COMT haplotype or the combined OPRM1/COMT genotype. These results are shown in Tables $3 \& 4$. One male Asian patient with an opioid requirement of $122.6 \mathrm{mg} / 48 \mathrm{~h}$ was identified as an outlier (Grubb's test, $\mathrm{p}<0.05$ ). This patient with extremely high postoperative opioid consumption was found to be homozygote variant carrier of the OPRM1 rs1799971 SNP.

\section{Genetics versus Postoperative chronic pain}

Chronic thoracic pain occurred 3, 6 and 12 months after cardiac surgery, respectively, in 53 patients (42.1\%), 37 patients $(29.8 \%)$ and 23 patients (18.8\%). After stratification according to randomization group (only performed in the analysis at 3 months) and correction for age, the genetic variants (OPRM1 rs1799971, COMT rs4680, rs4818, rs4633, COMT haplotype and combined OPRM1 rs1799971/COMT rs4680 genotype) were not associated with the development of chronic thoracic pain at 3, 6 and 12 months after cardiac surgery.

\section{Genetics versus Preoperative \& postoperative thermal pain thresholds}

We have observed a trend between COMT haplotype with the pre-operative heat pain threshold $(\mathrm{p}=0.014)$ and cold pain threshold $(\mathrm{p}=0.045)$. Subjects with the LPS haplotype had the highest (mean: $44.9^{\circ} \mathrm{C}$ [standard error (SE): 0.32] heat pain thresholds followed by APS $\left(44.2^{\circ} \mathrm{C}\right.$ [0.39]) and HPS $\left(43.2^{\circ} \mathrm{C}\right.$ [0.62]). Individuals with the LPS haplotype were experiencing cold pain at a lower temperature compared with APS and HPS haplotype $\left(7.52^{\circ} \mathrm{C}[0.98]\right.$ vs $9.36^{\circ} \mathrm{C}[1.23]$ vs $11.7^{\circ} \mathrm{C}$ [1.94]). COMT rs 4818 was associated with cold detection threshold $(\mathrm{p}=0.041)$. The trend between COMT haplotype and cold pain threshold remained 3 days after surgery $(\mathrm{p}=0.043)$, but not after 12 months. Also a trend was observed with the heat detection threshold and the OPRMI SNP at 12 months after surgery $(\mathrm{p}=0.010)$. However, none of these findings passed significance after Bonferroni correction $(\mathrm{p}=0.0083)$. The results are displayed in Table $5 \mathrm{~A}-\mathrm{C}$. The mean and SE are corrected for the composite outcome age and reaction time. 
Research Article Matic, de Hoogd, de Wildt, Tibboel, Knibbe \& van Schaik

\begin{tabular}{|c|c|c|c|c|c|c|}
\hline Fentanyl group & $\mathrm{n}$ & Median & IQR & Lowest & Highest & $p$-value \\
\hline OPRM1 & & & & & & 0.75 \\
\hline G carrier & 17 & 25.1 & {$[20.2 ; 38.3]$} & 7.20 & 42.9 & \\
\hline OPRM1/COMT & & & & & & 0.67 \\
\hline $118 \mathrm{AA}$ and $472 \mathrm{~A}$ & 32 & 30.2 & {$[17.7 ; 38.4]$} & 5.10 & 51.2 & \\
\hline COMT haplotype & & & & & & $0.009^{\dagger}$ \\
\hline LPS & 35 & 30.1 & {$[19.1 ; 37.7]$} & 5.10 & 47.2 & \\
\hline APS & 19 & 34.6 & {$[26.2 ; 41.4]$} & 10.1 & 52.5 & \\
\hline HPS & 9 & 19.4 & {$[16.5 ; 23.0]$} & 13.2 & 33.4 & \\
\hline COMT rs4680 & & & & & & 0.14 \\
\hline COMT rs4818 & & & & & & 0.82 \\
\hline $408 C C$ & 25 & 30.3 & {$[18.7 ; 37.9]$} & 10.1 & 51.2 & \\
\hline $408 C G$ & 24 & 32.6 & {$[18.9 ; 40.3]$} & 5.10 & 52.5 & \\
\hline 408GG & 14 & 26.9 & {$[19.2 ; 35.6]$} & 8.70 & 47.2 & \\
\hline COMT rs4633 & & & & & & 0.14 \\
\hline $186 C C$ & 17 & 28.5 & {$[20.3 ; 36.3]$} & 8.70 & 52.5 & \\
\hline $186 C \mathrm{CT}$ & 30 & 26.1 & {$[16.9 ; 37.8]$} & 5.10 & 43.0 & \\
\hline 186TT & 16 & 34.5 & {$[27.2 ; 41.1]$} & 10.1 & 51.2 & \\
\hline Remifentanil group & $\mathbf{n}$ & Median & IQR & Lowest & Highest & $p$-value \\
\hline OPRM1 & & & & & & 0.25 \\
\hline APS & 23 & 28.7 & {$[19.2 ; 39.9]$} & 5.40 & 63.8 & \\
\hline HPS & 8 & 38.3 & {$[29.3 ; 41.6]$} & 26.0 & 49.3 & \\
\hline COMT rs4680 & & & & & & 0.66 \\
\hline $472 \mathrm{GG}$ & 13 & 36.4 & {$[20.3 ; 41.2]$} & 8.60 & 55.0 & \\
\hline $472 \mathrm{GA}$ & 33 & 36.8 & {$[26.2 ; 43.5]$} & 11.6 & 55.0 & \\
\hline 472AA & 16 & 31.3 & {$[24.9 ; 39.5]$} & 5.40 & 63.8 & \\
\hline COMT rs4818 & & & & & & 0.92 \\
\hline $408 \mathrm{CC}$ & 24 & 34.8 & {$[25.5 ; 40.7]$} & 5.40 & 63.8 & \\
\hline $408 C G$ & 33 & 34.3 & {$[23.1 ; 43.5]$} & 8.60 & 55.0 & \\
\hline 408GG & 5 & 36.4 & {$[30.4 ; 41.5]$} & 29.4 & 44.0 & \\
\hline COMT rs4633 & & & & & & 0.66 \\
\hline $186 C C$ & 13 & 36.4 & {$[20.3 ; 41.2]$} & 8.60 & 55.0 & \\
\hline $186 \mathrm{CT}$ & 33 & 36.8 & {$[26.2 ; 43.5]$} & 11.6 & 55.0 & \\
\hline $186 \mathrm{TT}$ & 16 & 31.3 & {$[24.9 ; 39.5]$} & 5.40 & 63.8 & \\
\hline
\end{tabular}


Table 4. Morphine-equivalent requirements until $48 \mathrm{~h}$ after cardiac surgery.

\begin{tabular}{|c|c|c|c|c|c|c|}
\hline Fentanyl group & $\mathrm{n}$ & Median & IQR & Lowest & Highest & p-value \\
\hline OPRM1 & & & & & & 0.99 \\
\hline AA & 46 & 39.1 & {$[25.5 ; 52.6]$} & 5.10 & 77.5 & \\
\hline $\mathrm{G}$ carrier & 17 & 35.1 & {$[25.9 ; 54.6]$} & 8.10 & 82.9 & \\
\hline OPRM1/COMT & & & & & & 0.17 \\
\hline 118AA and $472 \mathrm{~A}$ & 32 & 34.8 & {$[19.8 ; 50.3]$} & 5.10 & 76.2 & \\
\hline $118 \mathrm{G}$ and/or $472 \mathrm{GG}$ & 31 & 41.0 & {$[27.9 ; 58.1]$} & 8.10 & 82.9 & \\
\hline LPS & 35 & 41.0 & {$[27.9 ; 58.1]$} & 5.10 & 69.8 & \\
\hline APS & 19 & 43.1 & {$[31.8 ; 56.7]$} & 16.0 & 82.9 & \\
\hline HPS & 9 & 23.2 & {$[18.7 ; 34.2]$} & 16.3 & 35.1 & \\
\hline COMT rs4680 & & & & & & 0.27 \\
\hline $472 \mathrm{GG}$ & 17 & 41.0 & {$[30.9 ; 60.7]$} & 8.70 & 77.5 & \\
\hline $408 C G$ & 24 & 46.8 & {$[25.8 ; 59.7]$} & 5.10 & 77.5 & \\
\hline 408GG & 14 & 40.6 & {$[33.1 ; 51.9]$} & 8.70 & 67.2 & \\
\hline COMT rs4633 & & & & & & 0.27 \\
\hline $186 C C$ & 17 & 41.0 & {$[30.9 ; 60.7]$} & 8.70 & 77.5 & \\
\hline $186 C \mathrm{CT}$ & 30 & 34.8 & {$[19.0 ; 52.8]$} & 5.10 & 69.8 & \\
\hline $186 T T$ & 16 & 41.1 & {$[32.0 ; 51.1]$} & 16.0 & 82.9 & \\
\hline Remifentanil group & $\mathrm{n}$ & Median & IQR & Lowest & Highest & p-value \\
\hline OPRM1 & & & & & & 0.25 \\
\hline AA & 49 & 46.5 & {$[33.8 ; 54.9]$} & 18.0 & 98.3 & \\
\hline G carrier & 13 & 59.2 & {$[34.7 ; 71.8]$} & 11.6 & 122.6 & \\
\hline HPS & 8 & 54.1 & {$[46.9 ; 60.4]$} & 26.0 & 69.3 & \\
\hline COMT rs4680 & & & & & & 0.82 \\
\hline $472 \mathrm{GG}$ & 13 & 46.4 & {$[37.5 ; 51.4]$} & 18.6 & 75.0 & \\
\hline $472 \mathrm{GA}$ & 33 & 47.1 & {$[33.7 ; 60.8]$} & 11.6 & 98.3 & \\
\hline $472 \mathrm{AA}$ & 16 & 35.2 & {$[30.0 ; 63.2]$} & 18.0 & 122.6 & \\
\hline COMT rs4818 & & & & & & 0.81 \\
\hline $408 C C$ & 24 & 47.7 & {$[33.8 ; 60.4]$} & 18.0 & 122.6 & \\
\hline $408 C G$ & 33 & 44.3 & {$[33.7 ; 60.0]$} & 11.6 & 98.3 & \\
\hline 408GG & 5 & 46.4 & {$[36.7 ; 50.1]$} & 29.4 & 51.3 & \\
\hline COMT rs4633 & & & & & & 0.82 \\
\hline $186 C C$ & 13 & 46.4 & {$[37.5 ; 51.3]$} & 18.6 & 75.0 & \\
\hline $186 C T$ & 33 & 47.1 & {$[33.7 ; 60.8]$} & 11.6 & 98.3 & \\
\hline $186 \mathrm{TT}$ & 16 & 35.2 & {$[30.0 ; 63.2]$} & 18.0 & 122.6 & \\
\hline
\end{tabular}




\begin{tabular}{|c|c|c|c|c|c|c|c|c|}
\hline Genotype & $n$ & $\begin{array}{l}\text { Heat detection } \\
\text { threshold }\left({ }^{\circ} \mathrm{C}\right)\end{array}$ & $n$ & $\begin{array}{l}\text { Cold detection } \\
\text { threshold }\left({ }^{\circ} \mathrm{C}\right)\end{array}$ & $n$ & $\begin{array}{l}\text { Heat pain threshold } \\
\left({ }^{\circ} \mathrm{C}\right)\end{array}$ & $\mathrm{n}$ & $\begin{array}{l}\text { Cold pain threshold } \\
\left({ }^{\circ} \mathrm{C}\right)\end{array}$ \\
\hline \multicolumn{9}{|l|}{ OPRM1 } \\
\hline $118 \mathrm{AA}$ & 92 & $36.3(0.22)$ & 94 & $29.7(0.13)$ & 95 & $44.4(0.27)$ & 95 & $8.40(0.83)$ \\
\hline $118 \mathrm{G}$ allele & 29 & $36.2(0.40)$ & 29 & $29.6(0.24)$ & 30 & $44.3(0.48)$ & 30 & $9.68(1.48)$ \\
\hline$p$-value ${ }^{\dagger}$ & & 0.82 & & 0.89 & & 0.81 & & 0.45 \\
\hline \multicolumn{9}{|l|}{ COMT rs4680 } \\
\hline $472 \mathrm{GG}$ & 28 & $36.4(0.41)$ & 30 & $29.3(0.23)$ & 30 & $44.7(0.48)$ & 30 & $8.80(1.49)$ \\
\hline $472 \mathrm{GA}$ & 61 & $36.2(0.28)$ & 61 & $29.7(0.16)$ & 63 & $44.4(0.33)$ & 63 & $8.44(1.02)$ \\
\hline 472AA & 32 & $36.3(0.38)$ & 32 & $29.9(0.22)$ & 32 & $44.2(0.46)$ & 32 & $9.14(1.44)$ \\
\hline$p$-value ${ }^{\dagger}$ & & 0.87 & & 0.10 & & 0.51 & & 0.87 \\
\hline \multicolumn{9}{|l|}{ СОMT rs4818 } \\
\hline $408 \mathrm{CC}$ & 49 & $36.0(0.31)$ & 48 & $29.9(0.18)$ & 49 & $43.9(0.37)$ & 49 & $10.0(1.15)$ \\
\hline $408 C G$ & 55 & $36.4(0.29)$ & 56 & $29.7(0.17)$ & 57 & $44.6(0.34)$ & 57 & $7.80(1.07)$ \\
\hline 408GG & 17 & $36.8(0.52)$ & 19 & $29.0(0.28)$ & 19 & $45.1(0.59)$ & 19 & $8.04(1.85)$ \\
\hline$p$-value ${ }^{\dagger}$ & & 0.16 & & 0.014 & & 0.051 & & 0.22 \\
\hline \multicolumn{9}{|l|}{ COMT rs4633 } \\
\hline $186 C C$ & 28 & $36.4(0.41)$ & 30 & $29.3(0.23)$ & 30 & $44.7(0.48)$ & 30 & $8.80(1.49)$ \\
\hline $186 C \mathrm{CT}$ & 61 & $36.2(0.28)$ & 61 & $29.7(0.16)$ & 63 & $44.4(0.33)$ & 63 & $8.44(1.02)$ \\
\hline $186 \mathrm{TT}$ & 32 & $36.3(0.38)$ & 32 & $29.9(0.22)$ & 32 & $44.2(0.46)$ & 32 & $9.14(1.44)$ \\
\hline$p$-value ${ }^{\dagger}$ & & 0.87 & & 0.10 & & 0.51 & & 0.87 \\
\hline \multicolumn{9}{|l|}{ COMT haplotype } \\
\hline LPS & 62 & $36.5(0.27)$ & 65 & $29.4(0.15)$ & 66 & $44.9(0.32)$ & 66 & $7.52(0.98)$ \\
\hline APS & 42 & $36.2(0.33)$ & 42 & $29.9(0.19)$ & 42 & $44.2(0.39)$ & 42 & $9.36(1.23)$ \\
\hline HPS & 17 & $35.4(0.52)$ & 16 & $29.9(0.31)$ & 17 & $43.2(0.62)$ & 17 & $11.7(1.94)$ \\
\hline$p$-value ${ }^{\dagger}$ & & 0.058 & & 0.041 & & 0.014 & & 0.045 \\
\hline \multicolumn{9}{|l|}{ OPRM1/COMT } \\
\hline $118 \mathrm{AA}$ and $158 \mathrm{Met}$ & 67 & $36.2(0.26)$ & 67 & $29.7(0.15)$ & 68 & $44.3(0.32)$ & 68 & $8.20(0.97)$ \\
\hline $118 \mathrm{G}$ and $/$ or $158 \mathrm{Val} / \mathrm{Val}$ & 54 & $36.3(0.29)$ & 56 & $29.5(0.17)$ & 57 & $44.5(0.35)$ & 57 & $9.31(1.07)$ \\
\hline$p$-value ${ }^{\dagger}$ & & 0.75 & & 0.37 & & 0.63 & & 0.45 \\
\hline
\end{tabular}

\section{Discussion}

In an effort to assess the potential influence of OPRM1 and COMT genetic variants on postoperative acute, chronic and experimental (thermal) pain, 125 cardiac surgery patients were genotyped and analyzed. We found that the COMT HPS haplotype was related with decreased postsurgical morphine requirement during the first $24 \mathrm{~h}$. This effect was only found in individuals that were randomized to intraoperative fentanyl, but not in the remifentanil group. Additionally, a trend was found between the COMT haplotype with thermal pain, which was not significant after Bonferroni correction.

The observed trend between COMT haplotype and thermal pain points toward increased pain sensitivity reflected by increased heat pain at lower temperatures and increased cold pain at higher temperatures. Increased pain responsiveness in HPS haplotype carriers is in line with initial literature in 202 healthy female volunteers with mixed racial background (85\% European-Americans) on multiple pain evoking stimuli, with only thermal pain significantly associated [36]. In contrast, in another mixed population (European-Americans, African-Americans, Asian-Americans and Hispanics) of healthy subjects, no effect of the COMT haplotype was observed on thermal pain. In the latter study thermal pain was assessed via another method (briefly induced cold and heat pain) compared with the previous study [37], which could have confounded the results. Additionally, no effect of the COMT predicted phenotype group could be observed on thermal pain sensitivity in 1000 female patients undergoing breast surgery for cancer [38]. Focusing on other methods of experimentally induced pain, a study in healthy Chinese males could not confirm the effect of the COMT haplotype on pain evoked by transcutaneous electrical accupoint stimulation [39]. These studies suggest that the effect of COMT haplotype on pain seems to differ between pain modalities and patients, in the last case either due to ethnic background or diseased versus healthy subjects.

In our study, patients in the fentanyl group $(n=62)$ with the HPS haplotype had lower postoperative morphine need compared with the APS haplotype, while individuals with the HPS haplotype showed a trend towards higher pain responsiveness to experimental heat pain. These intuitively opposite effects can be attributed to the correlation between the dopaminergic and endogenous opioid system, as shown in animal models [40,41]. Stimulation of the dopamine system, which is comparable with the decreased COMT activity seen with the HPS haplotype, causes a 


\begin{tabular}{|c|c|c|c|c|c|c|c|c|}
\hline Genotype & $\mathrm{n}$ & $\begin{array}{l}\text { Heat detection } \\
\text { threshold }\left({ }^{\circ} \mathrm{C}\right)\end{array}$ & $\mathrm{n}$ & $\begin{array}{l}\text { Cold detection } \\
\text { threshold }\left({ }^{\circ} \mathrm{C}\right)\end{array}$ & $\mathrm{n}$ & $\begin{array}{l}\text { Heat pain threshold } \\
\left({ }^{\circ} \mathrm{C}\right)\end{array}$ & $n$ & $\begin{array}{l}\text { Cold pain threshold } \\
\left({ }^{\circ} \mathrm{C}\right)\end{array}$ \\
\hline \multicolumn{9}{|l|}{ OPRM1 } \\
\hline $118 \mathrm{AA}$ & 93 & $36.0(2.22)$ & 91 & $29.5(0.17)$ & 93 & $43.5(0.29)$ & 93 & $10.1(0.92)$ \\
\hline 118G allele & 30 & $36.2(2.40)$ & 30 & $29.5(0.30)$ & 30 & $43.0(0.52)$ & 30 & $12.5(1.63)$ \\
\hline$p$-value ${ }^{\dagger}$ & & 0.87 & & 0.90 & & 0.42 & & 0.21 \\
\hline \multicolumn{9}{|l|}{ COMT rs4680 } \\
\hline $472 \mathrm{GG}$ & 29 & $36.7(0.42)$ & 29 & $29.3(0.31)$ & 29 & $43.7(0.53)$ & 29 & $10.3(1.67)$ \\
\hline $472 \mathrm{GA}$ & 62 & $35.7(0.28)$ & 60 & $29.7(0.21)$ & 63 & $43.2(0.36)$ & 63 & $10.8(1.13)$ \\
\hline $472 \mathrm{AA}$ & 31 & $36.1(0.40)$ & 31 & $29.3(0.30)$ & 31 & $43.3(0.51)$ & 31 & $10.8(1.61)$ \\
\hline $\mathrm{p}$-value ${ }^{\dagger}$ & & 0.31 & & 0.96 & & 0.56 & & 0.85 \\
\hline \multicolumn{9}{|l|}{ COMT rs4818 } \\
\hline $408 C C$ & 47 & $35.9(0.33)$ & 47 & $29.5(0.24)$ & 48 & $42.9(0.41)$ & 48 & $11.8(1.28)$ \\
\hline $408 C G$ & 56 & $35.9(0.30)$ & 56 & $29.5(0.22)$ & 56 & $43.4(0.38)$ & 56 & $10.5(1.19)$ \\
\hline 408GG & 19 & $36.9(0.51)$ & 19 & $29.4(0.38)$ & 19 & $44.1(0.65)$ & 19 & $8.33(2.04)$ \\
\hline $\mathrm{p}$-value ${ }^{\dagger}$ & & 0.17 & & 0.96 & & 0.13 & & 0.16 \\
\hline \multicolumn{9}{|l|}{ COMT rs4633 } \\
\hline $186 C C$ & 29 & $36.7(0.42)$ & 29 & $29.3(0.31)$ & 29 & $43.7(0.53)$ & 29 & $10.3(1.67)$ \\
\hline $186 C \mathrm{CT}$ & 62 & $35.7(0.28)$ & 61 & $29.7(0.21)$ & 63 & $43.2(0.36)$ & 63 & $10.8(1.13)$ \\
\hline $186 \mathrm{TT}$ & 31 & $36.1(0.40)$ & 31 & $29.3(0.30)$ & 31 & $43.3(0.51)$ & 31 & $10.8(1.61)$ \\
\hline$p$-value ${ }^{\dagger}$ & & 0.31 & & 0.97 & & 0.56 & & 0.85 \\
\hline \multicolumn{9}{|l|}{ COMT haplotype } \\
\hline LPS & 66 & $36.1(0.28)$ & 66 & $29.6(0.20)$ & 66 & $43.7(0.35)$ & 66 & $9.29(1.09)$ \\
\hline APS & 37 & $36.3(0.37)$ & 40 & $29.3(0.26)$ & 40 & $43.2(0.44)$ & 40 & $11.7(1.40)$ \\
\hline HPS & 16 & $35.5(0.57)$ & 16 & $29.8(0.41)$ & 17 & $42.4(0.68)$ & 17 & $13.7(2.14)$ \\
\hline $\mathrm{p}_{\text {-value }}^{\dagger}$ & & 0.54 & & 0.96 & & 0.086 & & 0.043 \\
\hline \multicolumn{9}{|l|}{ OPRM1/COMT } \\
\hline $118 \mathrm{AA}$ and $158 \mathrm{Met}$ & 65 & $35.8(0.28)$ & 66 & $29.6(0.20)$ & 67 & $43.2(0.35)$ & 67 & $10.1(1.09)$ \\
\hline $118 \mathrm{G}$ and/or $158 \mathrm{Val} / \mathrm{Val}$ & 54 & $36.4(0.31)$ & 56 & $29.4(0.22)$ & 56 & $43.4(0.38)$ & 56 & $11.4(1.19)$ \\
\hline$p$-value ${ }^{\dagger}$ & & 0.17 & & 0.48 & & 0.70 & & 0.44 \\
\hline
\end{tabular}

decrease in the levels of endogenous peptides [40]. This decline leads to a compensatory rise in mu-opioid receptor expression, meaning that with the HPS haplotype there is less endogenous substrate to alleviate pain, but more receptors available for increased binding when exposed to exogenous opioids. Although our findings on morphine consumption are in line with the biological plausibility for the HPS and APS haplotype, we did not observe a higher postoperative consumption in the LPS group compared with the APS group. Also studies from literature on the direction of the effect of these haplotypes are inconclusive. For example, other studies found that individuals with the APS haplotype required the lowest morphine need [42,43]. Also opposite to our findings, a study in Han Chinese patients found higher postoperative fentanyl requirement after radical gastrectomy with the HPS haplotype [44].

Although in our cohort patients with the LPS haplotype indeed had higher morphine consumption compared with the HPS haplotype, the difference was not significant. As described in our method section the possible COMT haplotype outcomes (LPS/LPS, LPS/APS, APS/APS, APS/HPS and LPS/HPS) have been converted into three possible haplotype outcomes. This could have confounded the association. Unfortunately, our study cohort size was insufficient to perform the six haplotype outcomes separately in the analysis. Besides, we have decreased this size even further by the performed stratification (fentanyl vs remifentanil) of our cohort.

Interestingly, the COMT genetic effect on postoperative opioid demand was only observed in the fentanyl randomized patients and not in the remifentanil group. It could be that due to the considerable shorter half-life of remifentanil (3-10 min) compared with fentanyl (1-4 h) the mu-opioid receptor gets desensitized. This desensitization can omit the COMT haplotype effect of differences in mu-opioid receptor expression as a consequence of the genetic altered COMT activity. Remifentanil is also associated with opioid-induced hyperalgesia [45], probably due to its effect on the N-methyl-D-aspartate receptor [46]. It has been hypothesized that signaling of this N-methyl-Daspartate receptor may lead to opioid induced hyperalgesia [47]. This could also be the explanation of the increased morphine consumption directly after surgery and increased postoperative pain 3 months after surgery that were reported in the primary analysis of this study [25].

In this cohort, we were unable to confirm the OPRM1 $118 \mathrm{~A}>\mathrm{G}$ effect on thermal, postoperative acute and chronic pain. Although we have observed a trend with the heat detection threshold 12 months after surgery, 


\begin{tabular}{|c|c|c|c|c|c|c|c|c|}
\hline Genotype & $n$ & $\begin{array}{l}\text { Heat detection } \\
\text { threshold }\left({ }^{\circ} \mathrm{C}\right)\end{array}$ & $n$ & $\begin{array}{l}\text { Cold detection } \\
\text { threshold }\left({ }^{\circ} \mathrm{C}\right)\end{array}$ & $n$ & $\begin{array}{l}\text { Heat pain threshold } \\
\left({ }^{\circ} \mathrm{C}\right)\end{array}$ & $n$ & $\begin{array}{l}\text { Cold pain threshold } \\
\left({ }^{\circ} \mathrm{C}\right)\end{array}$ \\
\hline \multicolumn{9}{|l|}{ OPRM1 } \\
\hline $118 \mathrm{AA}$ & 80 & $36.2(2.41)$ & 81 & $29.9(0.13)$ & 83 & $47.2(0.33)$ & 83 & $8.80(0.99)$ \\
\hline $118 \mathrm{G}$ allele & 28 & $35.1(1.20)$ & 27 & $30.0(0.23)$ & 28 & $46.2(0.58)$ & 28 & $10.2(1.72)$ \\
\hline$p$-value ${ }^{\dagger}$ & & 0.010 & & 0.60 & & 0.13 & & 0.48 \\
\hline \multicolumn{9}{|l|}{ СОMT rs4680 } \\
\hline $472 \mathrm{GG}$ & 24 & $36.1(0.45)$ & 26 & $29.7(0.23)$ & 26 & $46.8(0.60)$ & 26 & $7.45(1.77)$ \\
\hline $472 \mathrm{GA}$ & 57 & $35.7(0.29)$ & 56 & $30.0(0.16)$ & 58 & $47.3(0.40)$ & 58 & $9.06(1.18)$ \\
\hline $472 \mathrm{AA}$ & 26 & $36.1(0.44)$ & 27 & $29.9(0.23)$ & 27 & $46.5(0.59)$ & 27 & $11.0(1.73)$ \\
\hline$p$-value ${ }^{\dagger}$ & & 0.92 & & 0.61 & & 0.68 & & 0.16 \\
\hline \multicolumn{9}{|l|}{ СОMT rs4818 } \\
\hline $408 \mathrm{CC}$ & 42 & $35.8(0.34)$ & 41 & $29.9(0.18)$ & 43 & $46.5(0.47)$ & 43 & $10.8(1.36)$ \\
\hline $408 C G$ & 49 & $35.9(0.32)$ & 51 & $30.0(0.17)$ & 52 & $47.3(0.42)$ & 52 & $8.78(1.24)$ \\
\hline 408GG & 16 & $36.4(0.56)$ & 16 & $29.6(0.30)$ & 16 & $47.1(0.77)$ & 16 & $5.94(2.24)$ \\
\hline$p$-value ${ }^{\dagger}$ & & 0.41 & & 0.49 & & 0.32 & & 0.062 \\
\hline \multicolumn{9}{|l|}{ COMT rs4633 } \\
\hline $186 C C$ & 24 & $36.1(0.45)$ & 26 & $29.7(0.23)$ & 26 & $46.8(0.60)$ & 26 & $7.45(1.77)$ \\
\hline $186 C T$ & 57 & $35.7(0.29)$ & 56 & $30.0(0.16)$ & 58 & $47.3(0.40)$ & 58 & $9.06(1.18)$ \\
\hline $186 \mathrm{TT}$ & 26 & $36.2(0.44)$ & 27 & $29.9(0.23)$ & 27 & $46.5(0.59)$ & 27 & $11.0(1.73)$ \\
\hline$p$-value ${ }^{\dagger}$ & & 0.92 & & 0.61 & & 0.68 & & 0.16 \\
\hline \multicolumn{9}{|l|}{ COMT haplotype } \\
\hline LPS & 59 & $36.0(0.29)$ & 57 & $29.9(0.15)$ & 59 & $47.4(0.39)$ & 59 & $7.71(1.17)$ \\
\hline APS & 32 & $36.1(0.39)$ & 36 & $29.9(0.19)$ & 36 & $46.4(0.50)$ & 36 & $10.9(1.50)$ \\
\hline HPS & 16 & $35.1(0.55)$ & 15 & $30.0(0.29)$ & 15 & $47.0(0.77)$ & 16 & $10.5(2.24)$ \\
\hline$p$-value ${ }^{\dagger}$ & & 0.25 & & 0.87 & & 0.27 & & 0.13 \\
\hline \multicolumn{9}{|l|}{ OPRM1/COMT } \\
\hline $118 \mathrm{AA}$ and $158 \mathrm{Met}$ & 59 & $36.2(0.29)$ & 59 & $29.9(0.15)$ & 61 & $47.2(0.39)$ & 61 & $9.30(1.16)$ \\
\hline $118 \mathrm{G}$ and $/$ or $158 \mathrm{Val} / \mathrm{Val}$ & 48 & $35.6(0.32)$ & 50 & $29.9(0.17)$ & 50 & $46.6(0.43)$ & 50 & $8.98(1.28)$ \\
\hline$p$-value ${ }^{\dagger}$ & & 0.18 & & 0.74 & & 0.30 & & 0.85 \\
\hline
\end{tabular}

this was not significant after correction. Other studies investigating the effect of OPRM1 118A $>$ G genotype on experimental and postoperative pain showed inconclusive results, as recently was reviewed elsewhere [48]. A genegene interaction between OPRM1 and COMT could have biased the association with pain thresholds and opioid consumption [49]. However, this was not the case in our cohort, as no gene-gene OPRM1 COMT interaction has been observed.

A OPRM1 $118 \mathrm{~A}>\mathrm{G}$ gene-gender interaction has been described in the literature, with opposite effect found on pain between males and females [50-53]. These studies in general reported lower pain ratings among men that carry the $118 \mathrm{G}$ allele and higher pain ratings among woman with the $118 \mathrm{G}$ allele $[50,51,53]$, with the exception of one study that found the effect to be in the opposite direction [52]. Since our cohort existed of primarily males and we were consequently unable to illustrate an effect on thermal pain the effect might be less evident in males. Not acknowledging the interaction between this polymorphism with gender (and other clinical factors) might conceal the genotype effect on clinical outcomes and thus render its application for personalized pain treatment [54].

The gender-gene interaction is also described for COMT, namely in 143 healthy volunteers capsaicin-induced pain was solely higher among woman with the COMT HPS haplotype (low COMT activity) [55]. Decreased hepatic COMT activity has been reported in female individuals compared with males [56]. This gender difference may be related to estrogen levels, which has been supported by a study in rats illustrating downregulation of COMT activity by estrogen in the prefrontal cortex and the kidneys of the animals [57]. Due to the lower baseline levels in females they might be more prone to the decreased thermostability of the enzyme as a consequence of genetic variations in the COMT gene. In our primarily male cohort, we found an association between COMT haplotype and thermal pain thresholds. However, we were unable to assess if the effect of the COMT haplotype was larger in females due to the low inclusion rate of female subjects.

In this study, no genetic association between COMT and OPRM1 and the development of chronic pain was found. The role of the dopaminergic transmission in the development of chronic pain after surgery has been reviewed recently [58]. The COMT enzyme is one of the regulators of the dopaminergic transmission, by degradation of dopamine. As discussed in the previously mentioned review, studies have shown inconclusive results for this 
particular gene. One of the arguments that have been mentioned is that the development of chronic pain is complex and most likely caused by a combination of biological (e.g., genetic) factors, physical and social interaction [58]. The same argument is applicable for the OPRM1 genetic variant. We believe that the COMT haplotype analysis will not have a purpose as a standalone test in guiding pain therapy with opioids. However, this biomarker could be valuable in a multifactorial prediction model of opioid response and should be validated in an algorithm including other genetic and nongenetic factors.

A limitation of the study is that the remifentanil group also received fentanyl during surgery. In our design, we decided not to compare fentanyl with a study arm with remifentanil as single analgesic since it was expected that high doses of remifentanil would be needed in this painful and extended procedure. However, it is possible that the association between the genetic variants tested and postoperative morphine requirements in the remifentanil group is not found since this group received more intraoperative opioids.

\section{Conclusion}

In conclusion, we found the COMT haplotype to be associated with acute postoperative pain reflected by postoperative opioid consumption. Patients in the fentanyl group with the COMT HPS haplotype group required less postoperative morphine compared with the APS group. The COMT haplotype explained part of the variability in experienced postoperative pain directly after surgery, but not on the longer term after surgery.

\section{Summary points}

- COMT and OPRM1 are highly investigated genes in postoperative pain.

- Our study, in a cardiac surgery cohort, shows that the COMT haplotype (rs4680, rs4633, rs4818) was associated with acute postoperative pain in a subset of patients.

- COMT haplotype was not associated with chronic postoperative and experimental pain.

- No effect of OPRM1 rs1799971 variant was identified on postoperative acute, chronic and experimental pain.

- COMT haplotype will likely be valuable for the prediction of pain and response to opioids in a multifactorial model existing of biological, physical and social factors.

\section{Author contributions}

M Matic and S de Hoogd contributed equally to this research in collecting data, analyzing and preparing this manuscript. SN de Wildt, D Tibboel, CAJ Knibbe and RHN van Schaik studied the design and prepared the manuscript.

\section{Financial \& competing interests disclosure}

The authors have no relevant affiliations or financial involvement with any organization or entity with a financial interest in or financial conflict with the subject matter or materials discussed in the manuscript. This includes employment, consultancies, honoraria, stock ownership or options, expert testimony, grants or patents received or pending, or royalties.

No writing assistance was utilized in the production of this manuscript.

\section{Ethical conduct of research}

The authors state that they have obtained appropriate institutional review board approval and /or have followed the principles outlined in the Declaration of Helsinki for all human or animal experimental investigations. In addition, informed consent has been obtained from the participants involved in this study.

\section{References}

1. Mapplebeck JC, Beggs S, Salter MW. Sex differences in pain: a tale of two immune cells. Pain 157, S2-S6 (2016).

2. Tighe PJ, Riley JL, Fillingim RB. Sex differences in the incidence of severe pain events following surgery: a review of 333,000 pain scores. Pain Med. 15, 1390-1404 (2014).

3. Petrini L, Matthiesen ST, Arendt-Nielsen L. The effect of age and gender on pressure pain thresholds and suprathreshold stimuli. Perception 44, 587-596 (2015).

4. Meints SM, Miller MM, Hirsh AT. Differences in pain coping between Black and White Americans: a meta-analysis. J. Pain 17, 642-653 (2016).

5. Rahim-Williams B, Riley JL, Williams AKK, Fillingim RB. A quantitative review of ethnic group differences in experimental pain response: do biology, psychology, and culture matter? Pain Med. 13, 522-540 (2012). 
6. Leonard BE. Pain, depression and inflammation: are interconnected causative factors involved? Mod. Trends Pharmacopsychiatri. 30, 22-35 (2015).

7. Schug SA, Raymann A. Postoperative pain management of the obese patient. Best Pract. Res. Clin. Anaesthesiol. 25, 73-81 (2011).

8. Terry MJ, Moeschler SM, Hoelzer BC, Hooten WM. Pain catastrophizing and anxiety are associated with heat pain perception in a community sample of adults with chronic pain. Clin. J. Pain 32(10), 875-81 (2016).

-9. Angst MS, Phillips NG, Drover DR et al. Pain sensitivity and opioid analgesia: a pharmacogenomic twin study. Pain 153, 1397-1409 (2012).

10. Ahmedzai SH. Personalized medicine - one size fits one: tailoring pain therapy to individuals' needs. J. Pain Palliat. Care Pharmacother. 27, 83-85 (2013).

11. Clarke H, Katz J, Flor H, Rietschel M, Diehl SR, Seltzer Z. Genetics of chronic post-surgical pain: a crucial step toward personal pain medicine. Can. J. Anesth. 62, 294-303 (2015).

12. Trescot AM, Faynboym S. A review of the role of genetic testing in pain medicine. Pain Physician 17, 425-445 (2014).

13. Hwang IC, Park JY, Myung SK, Ahn HY, Fukuda K, Liao Q. OPRM1 A118G gene variant and postoperative opioid requirement a systematic review and meta-analysis. Anesthesiology 121, 825-834 (2014).

14. Ren ZY, Xu XQ, Bao YP et al. The impact of genetic variation on sensitivity to opioid analgesics in patients with postoperative pain: a systematic review and meta-analysis. Pain Physician 18, 131-152 (2015).

15. Tammimaki A, Mannisto PT. Catechol-O-methyltransferase gene polymorphism and chronic human pain: a systematic review and meta-analysis. Pharmacogenet. Genomics 22, 673-691 (2012).

16. Candiotti KA, Yang Z, Buric D et al. Catechol-o-methyltransferase polymorphisms predict opioid consumption in postoperative pain. Anesth. Analg. 119, 1194-1200 (2014).

17. Cargnin S, Magnani F, Viana M et al. An opposite-direction modulation of the COMT Val158Met polymorphism on the clinical response to intrathecal morphine and triptans. J. Pain 14, 1097-1106 (2013).

18. Rakvag TT, Ross JR, Sato H, Skorpen F, Kaasa S, Klepstad P. Genetic variation in the catechol-O-methyltransferase (COMT) gene and morphine requirements in cancer patients with pain. Mol. Pain 4, 64 (2008).

19. Tan EC, Lim EC, Ocampo CE, Allen JC, Sng BL, Sia AT. Common variants of catechol-O-methyltransferase influence patient-controlled analgesia usage and postoperative pain in patients undergoing total hysterectomy. Pharmacogenomics J. 16(2), 186-92 (2016).

20. Diatchenko L, Slade GD, Nackley AG et al. Genetic basis for individual variations in pain perception and the development of a chronic pain condition. Hum. Mol. Genet. 14, 135-143 (2005).

21. Nackley AG, Tan KS, Fecho K, Flood P, Diatchenko L, Maixner W. Catechol-O-methyltransferase inhibition increases pain sensitivity through activation of both beta(2)- and beta(3)-adrenergic receptors. Pain 128, 199-208 (2007).

22. Zeng BY, Jolkkonen J, Jenner P, Marsden CD. Chronic L-dopa treatment differentially regulates gene-expression of glutamate-decarboxylase, preproenkephalin and preprotachykinin in the striatum of 6-hydroxydopamine-lesioned rat. Neuroscience 66, 19-28 (1995).

23. Lotta T, Vidgren J, Tilgmann C et al. Kinetics of human soluble and membrane-bound catechol O-methyltransferase: a revised mechanism and description of the thermolabile variant of the enzyme. Biochemistry 34, 4202-4210 (1995).

24. Zubieta JK, Heitzeg MM, Smith YR et al. COMT val(158)met genotype affects mu-opioid neurotransmitter responses to a pain stressor. Science 299, 1240-1243 (2003).

25. de Hoogd S, Ahlers S, van Dongen EPA et al. Randomized controlled trial on the influence of intraoperative remifentanil versus fentanyl on acute and chronic pain after cardiac surgery. Pain Pract. 18, 443-451 (2018).

26. de Hoogd S, Ahlers SJ, van Dongen EP, Tibboel D, Dahan A, Knibbe CA. Remifentanil versus fentanyl during cardiac surgery on the incidence of chronic thoracic pain (REFLECT): study protocol for a randomized controlled trial. Trials 15, 466 (2014).

27. de Hoogd S, Valkenburg AJ, van Dongen EPA et al. Short- and long-term impact of remifentanil on thermal detection and pain thresholds after cardiac surgery: a randomised controlled trial. Eur. J. Anaesthesiol. 36, 32-39 (2019).

28. Ahlers SJ, van Gulik L, van Dongen EP et al. Efficacy of an intravenous bolus of morphine 2.5 versus morphine $7.5 \mathrm{mg}$ for procedural pain relief in postoperative cardiothoracic patients in the intensive care unit: a randomised double-blind controlled trial. Anaesth. Intensive Care 40, 417-426 (2012).

29. van Gulik L, Janssen LI, Ahlers SJ et al. Risk factors for chronic thoracic pain after cardiac surgery via sternotomy. Eur. J. Cardiothorac. Surg. 40, 1309-1313 (2011).

30. Electronic Medicines Compendium (eMC). Summary of product characteristics - OxyContin $5 \mathrm{mg}, 10 \mathrm{mg}, 15 \mathrm{mg}, 20 \mathrm{mg}, 30 \mathrm{mg}$, $40 \mathrm{mg}, 60 \mathrm{mg}, 80 \mathrm{mg}$ and $120 \mathrm{mg}$ prolonged release tablets. www.medicines.org.uk/emc/medicine/29384

31. Electronic Medicines Compendium (eMC). Summary of product characteristics - tramadol $50 \mathrm{mg}$ capsules. www.medicines.org.uk/emc/medicine/24675 
32. Nackley AG, Shabalina SA, Tchivileva IE et al. Human catechol-O-methyltransferase haplotypes modulate protein expression by altering mRNA secondary structure. Science 314, 1930-1933 (2006).

33. Matic M, Simons SHP, van Lingen RA et al. Rescue morphine in mechanically ventilated newborns associated with combined OPRM1 and COMT genotype. Pharmacogenomics 15, 1287-1295 (2014).

34. Reyes-Gibby CC, Shete S, Rakvag T et al. Exploring joint effects of genes and the clinical efficacy of morphine for cancer pain: OPRM1 and COMT gene. Pain 130, 25-30 (2007).

35. van Gulik L, Ahlers SJ, van de Garde EM et al. Remifentanil during cardiac surgery is associated with chronic thoracic pain 1 yr after sternotomy. Br. J. Anaesth. 109, 616-622 (2012).

36. Diatchenko L, Nackley AG, Slade GD et al. Catechol-O-methyltransferase gene polymorphisms are associated with multiple pain-evoking stimuli. Pain 125, 216-224 (2006).

37. Kim H, Mittal DP, Iadarola MJ, Dionne RA. Genetic predictors for acute experimental cold and heat pain sensitivity in humans. J. Med. Genet. 43, e40 (2006).

38. Kambur O, Kaunisto MA, Tikkanen E, Leal SM, Ripatti S, Kalso EA. Effect of catechol-o-methyltransferase-gene (COMT) variants on experimental and acute postoperative pain in 1,000 women undergoing surgery for breast cancer. Anesthesiology 119, 1422-1433 (2013).

39. Xiang X, Jiang Y, Ni Y et al. Catechol-O-methyltransferase polymorphisms do not play a significant role in pain perception in male Chinese Han population. Physiol. Genomics 44, 318-328 (2012).

40. George SR, Kertesz M. Met-enkephalin concentrations in striatum respond reciprocally to alterations in dopamine neurotransmission. Peptides 8, 487-492 (1987).

41. Steiner H, Gerfen CR. Role of dynorphin and enkephalin in the regulation of striatal output pathways and behavior. Exp. Brain Res. 123, 60-76 (1998).

42. De Gregori M, Garbin G, De Gregori S et al. Genetic variability at COMT but not at OPRM1 and UGT2B7 loci modulates morphine analgesic response in acute postoperative pain. Eur. J. Clin. Pharmacol. 69, 1651-1658 (2013).

43. Tan EC, Lim EC, Ocampo CE, Allen JC, Sng BL, Sia AT. Common variants of catechol-O-methyltransferase influence patient-controlled analgesia usage and postoperative pain in patients undergoing total hysterectomy. Pharmacogenomics J. 16, 186-192 (2016).

44. Zhang F, Tong J, Hu J et al. COMT gene haplotypes are closely associated with postoperative fentanyl dose in patients. Anesth. Analg. 120, 933-940 (2015).

45. Angst MS, Clark JD. Opioid-induced hyperalgesia: a qualitative systematic review. Anesthesiology 104, 570-587 (2006).

46. Fletcher D, Martinez V. Opioid-induced hyperalgesia in patients after surgery: a systematic review and a meta-analysis. Br. J. Anaesth. 112, 991-1004 (2014).

47. Guntz E, Dumont H, Roussel C et al. Effects of remifentanil on N-methyl-D-aspartate receptor: an electrophysiologic study in rat spinal cord. Anesthesiology 102, 1235-1241 (2005).

48. Gray K, Adhikary SD, Janicki P. Pharmacogenomics of analgesics in anesthesia practice: a current update of literature. J. Anaesthesiol. Clin. Pharmacol. 34, 155-160 (2018).

49. Khali H, Sereika SM, Dai F et al. OPRM1 and COMT gene-gene interaction is associated with postoperative pain and opioid consumption after orthopedic trauma. Biol. Res. Nurs. 19, 170-179 (2017).

50. Fillingim RB, Kaplan L, Staud R et al. The A118G single nucleotide polymorphism of the mu-opioid receptor gene (OPRMI) is associated with pressure pain sensitivity in humans. J. Pain 6, 159-167 (2005).

51. Hasvik E, Schistad EI, Grovle L, Haugen AJ, Roe C, Gjerstad J. Subjective health complaints in patients with lumbar radicular pain and disc herniation are associated with a sex-OPRM1 A118G polymorphism interaction: a prospective 1-year observational study. BMC Musculoskelet. Disord. 15(164), 161 (2014).

52. Linnstaedt SD, Hu JM, Bortsov AV et al. Mu-opioid receptor gene A118 G variants and persistent pain symptoms among men and women experiencing motor vehicle collision. J. Pain 16, 637-644 (2015).

53. Olsen MB, Jacobsen LM, Schistad EI et al. Pain intensity the first year after lumbar disc herniation is associated with the A118G polymorphism in the opioid receptor mu 1 gene: evidence of a sex and genotype interaction. J. Neurosci. 32, 9831-9834 (2012).

54. Belfer I, Young EE, Diatchenko L. Letting the gene out of the bottle OPRM1 interactions. Anesthesiology 121, 678-680 (2014).

55. Belfer I, Segall SK, Lariviere WR et al. Pain modality- and sex-specific effects of COMT genetic functional variants. Pain 154, 1368-1376 (2013).

56. Boudikova B, Szumlanski C, Maidak B, Weinshilboum R. Human liver catechol-O-methyltransferase pharmacogenetics. Clin. Pharmacol. Ther. 48, 381-389 (1990).

57. Schendzielorz N, Rysa A, Reenila I, Raasmaja A, Mannisto PT. Complex estrogenic regulation of catechol-O-methyltransferase (COMT) in rats. J. Physiol. Pharmacol. 62, 483-490 (2011).

58. van Reij RRI, Joosten EAJ, van den Hoogen NJ. Dopaminergic neurotransmission and genetic variation in chronification of post-surgical pain. Br. J. Anaesth. 123, 853-864 (2019). 